\title{
UN PASO DE MODERNIDAD Y SOLIDARIDAD EN LA REGULACIÓN DE TARJETAS DE DÉBITO Y CRÉDITO EN COSTA RICA
}

\author{
VELIA GOVAERE VICARIOLI \\ Ex ViceMinistra de Ministerio de Economia, Industria y Comercio (MEIC) \\ Observatorio de Comercio Exterior (OCEX) \\ Universidad Estatal a Distancia, Costa Rica \\ vgovaere@ocex.uned.ac.cr
}

\section{RESUMEN}

Este artículo analiza los principales elementos que integran la nueva legislación, a la luz de sus principales objetivos de información, divulgación y transparencia. Se explica la necesidad de equilibrar, vía reglamentaria, una relación contractual desigual entre emisores de tarjetas y tarjetahabientes. Se dimensiona el peso que este servicio tiene en una de las relaciones de servicio más álgidas para consumidores, comerciantes y pequeñas empresas (PYMES). Esta reflexión, enmarcada en el contexto de la crisis internacional, forma parte de la proyección universitaria del Observatorio de Comercio Exterior como una forma de vincular las condiciones de crédito con los esfuerzos comerciales, sobre todo de las pequeñas empresas. Se plantea esta novedosa regulación como un esfuerzo atinente por poner a nuestro país bajo el lineamiento de las tendencias internacionales, que incrementan el nivel de transparencia en el uso del mayor instrumento de financiamiento y de endeudamiento de nuestras poblaciones.

PALABRAS CLAVE: REGULACIÓN, REGLAMENTO, TARJETAS DE CRÉDITO, TARJETAS DE DÉBITO, EMISORES, CONSUMIDORES, TRANSPARENCIA.

\begin{abstract}
The main components of the new legislation and its main objectives in relation to information, publication, and transparency are analyzed. The need to balance out, through legal regulation, an uneven contractual relationship between credit card-issuing companies and cardholders is determined. The role played by credit cards services and companies in one of the most crucial and decisive business relations for consumers, entrepreneurs, and smaII and medium sized enterprises (SMEs) is weighed. In the context of an international crisis, the analysis proposed is part of the efforts of the UNED's Foreign Trade Observatory (OCEX), as a way to link the conditions of credit to the commercial efforts of small enterprises. This innovative legal regulation is proposed as a concerted effort to help Costa Rica meet international tendencies and standards intended to raise transparency levels in the use of today's most important instrument of financing and indebtedness in our countries.
\end{abstract}

KEYWORKS: ORDINANCE, REGULATION, CREDIT CARDS, DEBIT CARDS, ISSUING BANK, CONSUMERS, INFORMATION, TRANSPARENCY. 


\section{INTRODUCCIÓN}

"El pasado 8 de abril, el MEIC presentó, en la UNED, el nuevo Reglamento de Tarjetas de Crédito y Débito. Ningún lugar más idóneo que un paraninfo universitario que cumple así con su vocación de vincular academia con problemática nacional" (La Nación, abril 17, 2010).

La importancia de este tema puede vislumbrarse considerando que la correcta y adecuada regulación del mercado de tarjetas de crédito y de débito, afecta a millón y medio de familias de usuarios de costa a costa y frontera a frontera. Los valores manejados por estos instrumentos financieros son enormes y la deuda acumulada, con todo y su disminución frente a la crisis, representa el 2,6\% del PIB costarricense.

Voceros de entes emisores reconocieron públicamente, que se realizó, en algún momento, una repartición masiva de tarjetas de crédito sin evaluar la capacidad de pago de los clientes (Editorial (2010), La Nación, San José, Costa Rica, marzo 13). El último reporte trimestral del Ministerio de Economía, Industria y Comercio (MEIC) realizado en febrero de 2010 en materia de tarjetas, arrojó el inmenso número de diferentes tipos de tarjetas que se ofrecen en el país: 408 tipos de tarjetas, con tasas que oscilan entre el $20 \%$ y el 54\% anual. Para tener una idea del volumen de negocio y de endeudamiento que pesa sobre los hogares, se debe notar que sólo en enero de 2010 se aplicaron intereses por un monto de c61 893 millones (La Nación, marzo 13, 2010)

Por otra parte, las tarjetas de débito cobraron particular relieve e importancia desde que el Gobierno Central y sus entes descentralizados, municipalidades y otros entes públicos y privados han favorecido la utilización del pago de sus planillas a través de este medio.

El volumen de transacciones realizadas, la importancia que han adquirido en las economías familiares y en las finanzas de las instituciones emisoras son factores que demandaban una regulación apropiada de este instrumento de intermediación.

Este artículo analiza los principales elementos que integran la nueva legislación, a la luz de su principal objetivo, que busca equilibrar una desigual relación contractual entre los emisores de tarjetas y los tarjetahabientes, en una de las relaciones de servicio más álgidas para los consumidores, comerciantes y pequeñas empresas (PYMES) de nuestro país. Esta reflexión se hace todavía más oportuna desde la óptica de la dificultad implícita en el complejo proceso de nacimiento de esta regulación, que ha generado polémica pública y todavía posible litigio jurídico de parte de los emisores. Sin embargo, la desregulación atentaba principalmente contra el consumidor. Este esfuerzo regulatorio busca también poner a nuestro país al ritmo y bajo el lineamiento de las tendencias internacionales, que incrementan el nivel de transparencia en el uso del mayor instrumento de financiamiento y además de endeudamiento de nuestras poblaciones. El análisis de los elementos prioritarios que conforman la nueva regulación se enmarca también dentro de la proyección universitaria del Observatorio de Comercio Exterior de la UNED, como una forma de vincular las condiciones de crédito con los esfuerzos comerciales, sobre todo de las pequeñas empresas. Este es un factor más relevante en tanto existe la contradicción de una creciente dificultad por encontrar financiamiento de largo plazo y bajo costo y una creciente oferta de tarjetas que facilita el endeudamiento expedito a muy corto plazo y costos elevadísimos.

La participación de la autora, como viceministra de Economía y responsable del equipo de trabajo que elaboró el reglamento, permite la visión interna y la proyección política y social que esta reglamentación buscaba, así mismo da objetividad a los desafíos a los que se enfrentó esta normativa, no sólo desde el ángulo formal, sino 
también, tristemente, por medio de cabildeos y presiones políticas informales de parte de algunos emisores.

\section{Antecedentes}

La Ley 7472 de Promoción de la Competencia y Defensa Efectiva del Consumidor otorga al MEIC la capacidad de regular el mercado de tarjetas en lo concerniente a la defensa del consumidor. En función de esta capacidad se emitió un Reglamento Tarjetas de Crédito, en 2000, reformado posteriormente en 2003. En virtud de esa misma capacidad, el MEIC revisó la legislación existente $y$, ante sus carencias, se abocó, en 2009, a actualizar esa normativa, centrando su objetivo en ofrecer una mejora sustancial, exclusivamente en temas de información, divulgación y transparencia.

El primer reglamento de 2000, publicado vía decreto ejecutivo, constituía, al momento de su revisión, una regulación laxa y obsoleta, que requería un imperativo proceso de reforma, para responder a los desafíos de esta década. El punto de partida de esta evaluación radicaba en que dicha regulación solamente incluía cobertura exclusivamente para las tarjetas de crédito, sin ninguna referencia a transacciones a través de tarjetas de débito que tienen cada vez mayor volumen de vigencia. Por otra parte, esta regulación no contemplaba aspectos importantes de ser regulados, como abuso u hostigamiento en la cobranza, materia de seguros, comercio electrónico, información básica para el usuario, estándares armonizados en estados de cuenta, entre otros. La escasa cobertura de esta regulación hacía necesaria una revisión integral de la normativa de tarjetas en Costa Rica, dado que se consideró que dicho servicio se encontraba, en el entorno actual, prácticamente desregulado, en detrimento de todos sus usuarios.

Como parte integrante de las condiciones de endeudamiento de la población, la crisis eco- nómica de mediados de 2008, volvió concurrentemente perentoria la revisión del entorno regulatorio de este principal medio de crédito. Es ampliamente aceptado a nivel internacional, que el impacto negativo de la crisis en el endeudamiento de las familias se agravó también por la ausencia de regulaciones o regulaciones laxas, en materia de tarjetas de crédito y de débito, que no protegían en la forma debida a los consumidores. Es claro que, en medio de una crisis, no siempre es fácil cumplir fechas de pago. Para la pequeña empresa, es difícil, en particular, resistir la tentación y se aboca, con regularidad a resolver con ella flujos de caja.

Bajo el impacto de una crisis mundial, el contexto de los Estados Unidos y también, en general, en todo el hemisferio, muchos gobiernos se vieron obligados a introducir de urgencia nuevas reglas, incluso en ambientes políticos contenciosos, facilitando la modernización de la prestación del servicio de tarjetas de crédito.

Este fue el caso en Estados Unidos. Barack Obama había incluido, desde su campaña, un compromiso de regulación de tarjetas de crédito, como apoyo al consumidor y estímulo al comercio. En el primer trimestre de 2010 entró en vigencia la nueva regulación, que fue no solo el cumplimiento de una promesa de campaña, sino que además contó con el apoyo de todos los partidos. Estados Unidos no es el único caso de una regulación expedita en tiempos de esta crisis. Dentro del área centroamericana, El Salvador y Nicaragua han gestado en su seno respectivas legislaciones. En Panamá, donde hubo polémica en el legislativo, la nueva regulación costarricense fue tomada en consideración y tuvo su impacto positivo.

Costa Rica entendió la urgencia de regular el principal instrumento de endeudamiento de su población. La administración decidió tomar acción, como componente de su esfuerzo por ayudar a nuestra población a sobrellevar la crisis mundial, 
incluyendo una nueva regulación de tarjetas de crédito y débito, como parte integral de su "Plan Escudo". La visión subyacente a la actualización de la regulación de las tarjetas es verla como un componente complementario en la defensa de los consumidores. Se entiende su trascendencia porque a todos afecta su funcionamiento, sea al público en general como consumidor o parte de una planilla, a los comercios y a las pequeñas y medianas empresas.

\section{Reflexiones en torno al proceso de reforma}

El Ministerio de Economía, Industria y Comercio (MEIC) tiene bajo su tutela la defensa de los consumidores, de los comerciantes y el apoyo a la pequeña y mediana empresa. Esta delicada responsabilidad, aunada a la rectoría que le otorga la Ley n. 7472 en su artículo 41, motivó a esta institución a iniciar labores para la actualización y modernización del principal medio de pago de la población costarricense.

Los procesos modernos de reformas públicas comienzan por estudiar las mejores prácticas internacionales, sus impactos, sus beneficios y sus contradicciones y adaptarlos a la propia realidad nacional. Ese fue el modelo bajo el cual se basó el Ministerio de Economía. El MEIC involucró la participación activa de sus diversas direcciones, atendiendo así su propia diversidad, en una articulación de esfuerzos para obtener un resultado mancomunado de sus capacidades institucionales. Confluyeron así, insumos de la Dirección del Consumidor, Estudios Económicos, Competencia, Mejora Regulatoria y Asesoría Legal. Se tomaron como punto de partida, las mejores prácticas legales internacionales, bajo la luz de un estudio comparativo que incluyó la nueva legislación estadounidense, diversas legislaciones latinoamericanas y algunas europeas, como la legislación española. Con ese formato de análisis, el MEIC se abocó a elaborar un anteproyecto de actualización de la regulación de tarjetas, como parte de su compromiso con la debida protección a consumidores, PYMES y comerciantes.

Al tenor de un proceso de reforma moderno, vistos los problemas planteados por los mismos consumidores y usuarios, analizados los temas a la luz de la experiencia y el derecho comparado, a finales de 2009, se puso a disposición de una amplia consulta pública ciudadana, una propuesta de reglamento. Desde el 23 de octubre de 2009, fue puesta a disposición de todos los interesados una primera propuesta de "Reglamento de Tarjetas de Crédito y Débito". En la consulta pública participaron activamente tanto emisores como organizaciones de consumidores, comerciantes y público en general.

Se recibieron numerosas contribuciones, sugerencias, críticas y solicitudes que fueron analizadas, sopesadas e incluidas en lo pertinente, en un proceso que duró varios meses.

Resultado de un particularmente largo y complejo proceso, fue la publicación en La Gaceta del 30 de Marzo de 2010, en su alcance N.4, del Decreto Ejecutivo "Regulación de Tarjetas de Crédito y Débito", que le da vigencia a la nueva normativa.

\section{Los objetivos de la normativa}

El primer pilar del nuevo reglamento de tarjetas de crédito y débito se aboca a mejorar la calidad de información para el consumidor. Se procura que el consumidor disponga de información veraz, clara, uniforme y de fácil comprensión, que incida en una mejor decisión de consumo.

Se procuró así enfrentar el problema de que la mayoría de nuestra población hace uso de tarjetas, y muchas familias tienen varios tipos al mismo tiempo, de parte de diferentes emisores, con lo que sus deudas se acumulan, sin tener claridad ni de los plazos en que podrán saldar 
sus obligaciones, ni de la composición, cuando pagan, de la parte destinada al principal y la parte destinada a los intereses que adeudan. La diversidad de formatos de estados de cuenta dificultaba adicionalmente, una comprensión uniforme del estado de situación de los deudores y tampoco dejaba claro si los intereses actuales adeudados se aplican o no sobre intereses anteriores. De hecho, si se estudian los sistemas de composición de la deuda y la asignación de cobros de los emisores, se podría tener la inquietud de si aplica, en muchos casos, el sistema de capitalización de intereses. El nuevo reglamento prohíbe expresamente esta práctica y establece un modelo de estado de cuenta armonizado, que facilita la claridad del cobro de intereses y que estos no se acumulen a la deuda principal.

Así lo hace también la reciente regulación norteamericana. Como en Estados Unidos, el principal instrumento de nuestra normativa es la estandarización de la información contenida en los estados de cuenta. Se permite que cada ente emisor guarde su derecho al formato de imagen corporativa, pero se hace obligatorio tener un mismo formato de información de cuentas, con diferenciación, en cada pago mínimo, entre la parte que va al pago de la deuda principal y la consagrada a los intereses.

Esta estandarización obligaría también a que los pagos mínimos se ajusten de forma tal que se pueda cancelar la totalidad de la deuda en el tiempo estipulado. Se busca, de esta forma, ofrecer al usuario la información que le permita entender mejor las consecuencias de sus decisiones de consumo y saber cuándo concluye su deuda.

Un objetivo transversal de la nueva regulación ha sido equilibrar el peso contractual a favor del más débil. Es la tendencia jurídica moderna que no entiende como iguales a partes social y económicamente desiguales. Es el espíritu de nuestro ordenamiento jurídico, desde el código laboral hasta la ley de Inquilinato.

Vale aclarar que, a diferencia de la tendencia internacional, la nueva normativa -vía reglamento- no tiene la potestad de regular tasas y comisiones. La tendencia internacional ha regulado tasas para el tarjetahabiente y comisiones al comercio. Bajo el ámbito jurídico nacional se requiere de una Ley vía Poder Legislativo, para poder incluir una regulación de tasas al usuario y comisiones al comercio. Queda como deuda para futuro, la consolidación de una propuesta vía ley, que tenga viabilidad en nuestra Asamblea Legislativa.

\section{El cuerpo normativo del Reglamento de tarjetas}

La nueva regulación introdujo modificaciones que incorporaron elementos o mejoraron los ya existentes en el reglamento de 2000. Se cubrieron temas como acoso u hostigamiento para la cobranza, amortización, beneficios, comercio electrónico, contrato de emisión de tarjeta de débito, servicios accesorios, regulación de funcionamiento de tarjeta de débito, seguros y protección de datos personales.

Es de especial relevancia el conocimiento para el usuario de los principales rubros que componen esta nueva normativa. A continuación, se presenta una visión panorámica y sistémica de sus principales reglas:

1) En el campo de las definiciones, éstas se actualizan conforme a las nuevas prácticas y usos, agregando nuevos conceptos, lo que permite mayor claridad y transparencia para todos los sujetos de la regulación. Uno de los conceptos incluidos corresponde al abuso u hostigamiento en la cobranza, completamente novedoso en este contexto. 
2) Se mejoró la forma e interpretación de los contratos, promoviendo que sus condiciones generales sean suficientemente claras y precisas, a fin de no inducir en error a los consumidores. En caso de duda en la interpretación de las condiciones generales, se adopta el principio jurídico de protección especial a la parte contractual más débil, lo que implica que se resolverá a favor de los consumidores. Se aplica de esta forma el concepto de indubio pro consumidor. De igual manera y con la misma base jurídica, se utilizó el principio de las cargas probatorias dinámicas, que sitúan la carga de la prueba en quien está en mejor posición de llevarla a cabo, en este caso, los emisores.

3) Se regulan los contratos de tarjeta de débito, estableciendo la obligación para los emisores de brindar información sobre las transacciones que realiza el tarjetahabiente con el plástico, fundamentado en el derecho de información veraz y oportuna que tienen los consumidores.

4) Se reguló la libre contratación de los seguros. Según esto, los emisores no podrán exigir que los contratos de seguros de sus clientes estén predeterminados con una entidad aseguradora o intermediario de seguros. Cuando el emisor asuma una póliza colectiva, no podrá trasladar al tarjetahabiente los costos de las primas por concepto de ese seguro colectivo.

5) Se establecieron prohibiciones de condicionamiento de la contratación, inhibiendo el otorgamiento de tarjetas condicionadas a la contratación de los seguros o a cualquier otro servicio accesorio que ofrezcan. Esto faculta al tarjetahabiente a contratar libremente la póliza y otros servicios en cualquiera de las entidades que lo comercialicen. Esto se conoce bajo la figura de "ventas atadas", y constituye una de las principales quejas del usuario ante la Dirección de Apoyo al Consumidor del mismo MEIC.

6) Se establecen las condiciones bajo las cuales se pueden modificar los contratos. El emisor está obligado a notificar cambios por escrito, al tarjetahabiente y al fiador, el aviso de modificación del contrato. Se deberá prevenir al tarjetahabiente y al fiador (cuando éste se viera afectado) su capacidad de aceptar o no la modificación.

7) Se establecieron condiciones de armonización de los Estados de Cuenta:

a) En el caso de las tarjetas de crédito se estableció obligatoriedad de apegarse al modelo establecido en la normativa, que manda:

- Separar los saldos de principal e intereses.

- Suministrar las fórmulas de cálculo.

- Ajustar los pagos mínimos a derecho de manera que la deuda sea pagada en el plazo señalado.

- Separar la información correspondiente a otras líneas de crédito otorgadas en forma paralela al uso de la tarjeta de crédito y para las cuales prevalecen plazos y tasas de interés distintas de las pactadas contractualmente. Para éstas deberá presentarse un estado de cuenta separado, pudiendo hacerse en un mismo cuerpo documental, según Anexo del Reglamento.

- Cobrar como cargos por gestión de cobro, solamente aquellos que hayan sido expresamente autorizados por el tarjetahabiente y deberán incluirse de manera separada y detallada en el estado de cuenta. 
- El cargo de la comunicación o aviso no podrá exceder el costo de la tarifa del medio de comunicación elegido.

b) En el caso de la Tarjeta de Débito

- Se obliga a indicar la tasa pasiva y la forma de cálculo.

- A los tarjetahabientes de débito, los estados de cuenta le serán enviados al menos cada tres meses, pero no se podrá negar información actualizada al tarjetahabiente que lo solicite en cualquier momento. Este envío deberá realizarse por el medio de comunicación elegido por el tarjetahabiente. En iguales condiciones, el estado de cuenta deberá estar a disposición del tarjetahabiente en las oficinas o agencias del emisor.

8) Se regula el Derecho a la Protección de Datos personales de los tarjetahabientes bajo los siguientes lineamientos:

- $\quad$ Los usuarios de servicios financieros tienen derecho a la protección de los datos personales que las entidades financieras obtengan para la prestación de sus servicios.

- El tratamiento de los datos personales con fines distintos a los exceptuados según la Ley requerirá consentimiento expreso, libre e informado de los tarjetahabientes.

- Los usuarios de los servicios financieros tienen derecho a solicitar la rectificación de sus datos y, cuando proceda, a la cancelación de aquello que no corresponda a su situación actual.
9) Protección del patrimonio de los tarjetahabientes a través de la Banca Electrónica. Las entidades financieras deberán adoptar las medidas técnicas de seguridad específicas que requieren las transacciones realizadas con tarjetas de débito y de crédito por medios electrónicos.

10) Se regula el uso de los dispositivos para procesar transacciones. Esto quiere decir que los establecimientos comerciales deberán tener siempre a la vista de los consumidores las máquinas procesadoras de transacciones mediante tarjetas de crédito y débito, de forma tal que el tarjetahabiente no las pierda de vista durante la operación de pago. Los establecimientos en los que, por su naturaleza, los pagos se realicen en un lugar distinto de la caja, deberán contar con la tecnología adecuada para realizar con seguridad la operación de pago.

11) Se regula el cobro de comisiones de uso de los cajeros automáticos. Las entidades que operen cajeros automáticos deberán informar de manera previa, en las pantallas, el costo específico de cualquier transacción que demande, en ese momento el tarjetahabiente. El transitorio para este acápite es de 180 días, a fin de que los emisores puedan tomar las medidas técnicas pertinentes para la correcta implementación de esta disposición.

12) Se establece un régimen de responsabilidad. Esto implica que todas las entidades y participantes involucrados en el perfeccionamiento de las transacciones de tarjetas de crédito y débito, deben responder concurrente e independientemente de la existencia de culpa, frente al tarjetahabiente por cualquier daño que se le cause. Esto incluye los casos de sustracción de datos de seguridad, duplicación de la tarjeta o cobros de cargos no autorizados. 
13) Se establecen restricciones a las prácticas abusivas en las cobranzas. Las entidades financieras, abogados, gestores o agencias de cobranza, para llevar adelante las gestiones de cobro, deberán hacerlo directamente con el deudor y sus fiadores, es decir, las partes contractualmente relevantes. No se podrá realizar dicha gestión con personas ajenas a esta relación contractual. Tampoco podrán utilizar prácticas de acoso y hostigamiento para el cobro de las deudas. Se establece la posibilidad de cobrar solamente 3 llamadas en la gestión de cobro. Cualquier otro cargo deberá constar de forma separada en el Estado de Cuenta en concepto de gastos por gestión de cobro, a fin de garantizar transparencia al tarjetahabiente.

14) Se establece la obligatoriedad de devolución de documentos. Al término de la relación contractual el emisor deberá gestionar la devolución de los documentos que le fueron dados en garantía del crédito asociado a la tarjeta de crédito y ponerlos a disposición del cliente.

15) Se establece un régimen de Sanciones. Éstas se enmarcan bajo la Ley n. ${ }^{\circ} 7472$, relativa a derechos del consumidor, deberes del comerciante y publicidad (multas).

16) Se ampliaron los beneficios de servicio al cliente ofrecidos por los emisores, con la obligatoriedad de servicios de información y mayor responsabilidad frente a reclamos en términos de plazos. Se estableció la obligatoriedad de dar servicio de forma gratuita en lo que se refiere a información de productos, procedimientos, normativa, autoconsulta y estados de cuenta.

17) Se mejoró el sistema de cálculo en los Estados de Cuenta. Se introduce la prohibición expresa de cargos de intereses capitalizables, así como cargos por gestión de cobro no autorizados y la imposición de cargos no autorizados por sobregiros en las tarjetas de débito. En anexo, el reglamento muestra un "modelo" de la información y cómo debe presentarse el estado de cuenta.

18) Se mejoraron las condiciones de uso de las tarjetas durante los períodos de reclamo, haciendo prohibición de dificultar el uso de la tarjeta mientras dure el reclamo.

\section{Reflexiones sobre la Responsabilidad Social Empresarial}

Costa Rica se enorgullece de contar con instituciones financieras preocupadas por su Responsabilidad Social Empresarial (RSE). La banca estatal y privada costarricense realizan múltiples esfuerzos y loables iniciativas para orientar parte de sus ganancias hacia diversos proyectos de apoyo a las comunidades, educación, Pymes, medio ambiente y tantas otras formas de manifestación de compromiso con el mejoramiento de nuestra sociedad y de cumplimiento voluntario de su responsabilidad empresarial.

Cabe destacar, sin embargo, que la primera responsabilidad social de una empresa, más allá de acciones altruistas, comienza por asumir responsabilidades sociales en su propio giro de negocios, es decir, actuando de forma tal que sus clientes y usuarios tengan el mayor grado de satisfacción y transparencia.

Este mismo espíritu estuvo presente en la consulta pública del Reglamento donde el tema de discusión técnica fue solamente la mejor manera de llenar esos propósitos. Ambos sectores, gobierno y empresas financieras, tuvieron bajo la mira el bienestar de los mismos sujetos, sea como clientes o como ciudadanos. Es su satisfacción como tarjetahabientes o como consumidores lo que estaba en juego en este proceso. Es por ello, relevante destacar que este Reglamento representa, para los emisores, la oportunidad de aplicar la RSE precisamente en su propio giro 
de negocios, incrementando el nivel de información, divulgación y transparencia, para satisfacción de sus usuarios.

Como se dio a conocer por diferentes medios de prensa, este proceso se vio empañado por esfuerzos de cabildeo o presiones políticas de algunos emisores, al margen de los canales apropiados. En estos momentos el nuevo reglamento está ya vigente. Su futuro, sin embargo, todavía está sujeto a que las nuevas autoridades respondan a mociones y planteamientos a destiempo que han planteado algunos emisores, así como a amenazas abiertas de recursos de constitucionalidad, que, no obstante, no han visto todavía la luz. Uno de los mayores peligros que corre la nueva regulación es, precisamente, abrir el portillo para modificaciones parciales, aparentemente inocuas, como la suspensión propuesta, postergando para las calendas griegas el nuevo estado de cuenta, con lo que la nueva regulación perdería, de uno en uno, su peso.

\section{CONCLUSIONES}

Todos los cambios indicados previamente responden a elementos nuevos que no existían en la regulación de 2000, salvo excepciones puntuales. El estudio, sin embargo, de la legislación comparada, así como las quejas de los usuarios y el propio análisis del grupo multidisciplinario del MEIC permitieron introducir sustanciales mejoras en la regulación que estaba vigente.

Frente a una de las más importantes prestaciones ofrecidas por las instituciones financieras y uno de los servicios más álgidos para el consumidor, la normativa expuesta ha actualizado, mejorado e incrementado el nivel de transparencia y protección al usuario. Esta regulación busca el beneficio de ambas partes de una de las relaciones contractuales más decisivas de la vida social moderna.
En muchas partes los consumidores sentirán que la legislación es insuficiente y tendrán tal vez razón, pero era lo que la "cobija de la ley", en el momento de su elaboración y hasta ahora en el presente, permitía. Algunos emisores percibirán, en cambio, que se presentó un exceso en la regulación. Era de esperarse, en todas partes ocurre y Costa Rica no fue excepción. Pero la regulación era necesaria y en tiempos de crisis, solidaria.

No es de excluir, y más bien se esperaría en el futuro, que, conforme indican nuestros medios de comunicación: "se ponga en agenda, un análisis de la proporcionalidad y racionalidad del amplio espectro de diferentes tipos de intereses y comisiones que pagan tarjetahabientes y comercio" (Editorial (2010), La Nación, San José, Costa Rica, Marzo 13). Sería un paso adicional en la dirección correcta, para ayudar a las familias a resolver sus problemas financieros, agravados por la crisis.

En todo caso, este reglamento corresponde por su vocación de solidaridad a nuestro imaginario social y responde a la tendencia internacional. Como tal es un paso hacia la modernidad de nuestras instituciones y prácticas financieras.

\section{REFERENCIAS}

Central America Data (13 de noviembre, 2009). El Salvador: aprueban Ley de Tarjetas de Crédito. Recuperado el 2305-10 de: http://www.centralamericadata.com/es/ article/home/El_Salvador_aprueban_Ley_de_Tarjetas_de_Credito

La Nación (13 de marzo, 2010) Editorial: Un paso en la dirección correcta. Recuperado el 22-05-10 de: http://www. myvirtualpaper.com/doc/Nacion/la_nacion-13marzo2010/2010031302/1.html

La Nación (17 de abril, 2010) Editorial: Un desbalance permanente. Recuperado el 22-05-10 de: http://www.myvirtualpaper.com/doc/Nacion/la_nacion-17abril2010/20 10041702/40.html 
Ley n. 7472 (1995). Ley de Promoción de la Competencia y Defensa Efectiva del Consumidor. San José, Costa Rica. Recuperado el 23-05-10 de: http://www.tramites. go.cr/MarcoLegal/archivo/7472.pdf

Ministerio de Economía, Industria y Comercio (2010). Estudio comparativo de Tarjetas de Crédito. Recuperado el 23-0510 de: http://www.pymesdotcom.com/blog/2010/03/ estudio-tarjetas-credito\%E2\%80\%93febrero-2010/

Oficina de Prensa de la Casa Blanca (22 de febrero, 2010). Fact Sheet Reform to Protect American Credit Card Holders. President Obama signs Credit Card Accountability, Responsibility, and Disclosure Act. Washington DC, Estados Unidos. Recuperado el 23-05-10 de: http:// www.whitehouse.gov/the_press_office/Fact-SheetReforms-to-Protect-American-Credit-Card-Holders/

PaymentMedia (19 de febrero, 2010). Panamá: ley regula el mercado de tarjetas de crédito. Recuperado el 24-05-10 de: http://www.paymentmedia.com/scripts/templates/pagina_principal.asp?nota=contenidos_nuevos/ Medios\%20de\%20pago\%20elect/Tarjetas\%20Americ a\%20Lat/2010/Febrero/19_Panama_ley_tarjetas_de_ credito\&despliegue=desarrollo_noticia.asp

Reglamento de Tarjetas de Crédito y Débito, Decreto n. ${ }^{\circ}$ 35867, publicado en La Gaceta del 30 de Marzo de 2010, Alcance n. ${ }^{\circ}$ 4. Recuperado el 22-05-10 de: http:// meic.go.cr/esp2/informacion/decreto35867.asp

Recibido: 25-05-2010

Aceptado: 27-09-2010 